\title{
ScienceHumanities: Introduction
}

\section{James Castell, Keir Waddington \& Martin Willis}

The ScienceHumanities was founded at Cardiff University in 2016 to investigate the present and future challenges, functions and successes of collaborative research between the humanities and the sciences. It was led by the authors: Martin Willis and James Castell from the School of English, and Keir Waddington from the School of History, Archaeology and Religion. Over the course of 2016 the ScienceHumanities initiative focused on generating new discussions and ways of thinking within and beyond Cardiff. Leading international scholars across disparate humanities disciplines were invited to give seminars and public lectures, while we attended international events to discuss the nature and role of the ScienceHumanities. We held workshops and exhibitions investigating discrete forms of collaboration between the humanities and the sciences in order to build and develop new critical interactions between different fields of inquiry. In December 2016, we held a colloquium that brought together scholars from three continents and multiple disciplines to begin to offer initial definition to the ScienceHumanities and the role that it might play in our research future. This special issue of the Journal of Literature and Science arises from that colloquium and our thinking.

The ScienceHumanities is an ambitious attempt to think and rethink the relationships and the boundaries between the humanities and the sciences. Rather than rehearsing the familiar two cultures debates, we believe that the global challenges facing us now and in the future demand an urgent and rigorous reassessment of how we conceptualize disciplinary boundaries and the production of knowledge. It is for this reason that our term - ScienceHumanities - exists as a blended version of an earlier disciplinary binary. At its core, ScienceHumanities highlights the theoretical, political, and practical necessity of plural humanities approaches in place of the more singular disciplinary methodologies that continue to remain, in our view, more common. The term also positions the disparate disciplines of the sciences and the humanities in close proximity. This is intended to produce new perspectives on collaborative working, as well as to place emphasis on the importance of interrogating how such collaborations can function. Finally, the elision of the space between "science" and "humanities" is emblematic of the transdisciplinary nature of the ScienceHumanities; which works across and between the disciplines and their methodologies, seeking to find new knowledge in the interstices of their joining together rather than in their separate spheres. We extend each of these points in our own article, "ScienceHumanities: Theory, Politics, Practice" which opens the special issue.

Following our own prelude, the special issue is organized into three sections: Triangulations, Speculations, Practices. These three sub-titles capture the range of approaches taken by our contributors. Some, as the first section on Triangulations reveals, reconsider the relationship between the humanities and the sciences in specific and innovative ways. Others, in section two on Speculations, focus on how to attune the research in different humanities fields to the ScienceHumanities. In the third section on Practices, other contributors offer examples of where collaboration is at its most fruitful or most frustrating for all the disciplines involved. As suits such variety in approaches, the articles are written in different styles and with considerable variation 
in tone, register and even structure. One article provides the transcript of a correspondence between a humanist and scientist for example, while others respond in personal ways to disciplinary assumptions and hierarchies. By contrast, other articles draw on distinct disciplinary registers more commonly found in academic work, while a final grouping moves to and fro between the personal and the critically-distanced. All the articles, style aside, focus in engaging and productive ways with how we can define and work with the ScienceHumanities, either through addressing its concerns head-on or by reflecting implicitly on the core of ideas that have informed it. In the sub-sections below, we provide an outline of each article's contribution before concluding with brief reflections on the knowledge produced by them as a collective, finishing by assessing how that knowledge contributes to future directions for the ScienceHumanities.

\section{Triangulations}

In opening the special issue, literature and medicine scholar Catherine Belling argues for the centrality of the humanities in thinking about the relationship between the sciences, arts and humanities. Starting with a common binary, the opposition between the arts and the sciences, Belling contends that the humanities can create a more productive triangulation of knowledge. For Belling, the humanities have an important role in mediating between the arts and sciences. Through an examination of the kinds of assumptions made about different forms of knowledge-making and expertise, Belling argues that the humanities change the dynamic of more binary approaches. The humanities make clear that both art and science are not working within silos but instead that they are complex products of human knowledge responding to, and ethically aligned with, the natural world. It is, Belling concludes, the humanities and the methods of their analysis that remind us of this and enact it.

The second article extends these ideas by thinking about how triangulations between disciplines might occur. Here, literary scholars Anton Kirchhofer and Anna Auguscik map a history of the two cultures and see it as continuing far past C.P. Snow's initial intervention. Through an interrogation of media responses to the Anglophone science novel and debates surrounding climate change, their innovative solution to this challenge is to move away from current perceptions of the humanities by the sciences and vice versa. Instead, they argue for the importance of looking at how external observers view both disciplinary positions. This enables a fresh perspective that is not conditioned by the mutual observation that traditionally characterises debate. Their recognition that the humanities promote both a plurality of perspectives and a critical eye can tell us something about how the humanities can function within the ScienceHumanities. For Kirchhofer and Augusick, the humanities offer to the sciences new ways to represent themselves afresh to public audiences and to debate.

Where Belling and Kirchhofer and Augusick offer ways out of unproductive binaries, sociologist Des Fitzgerald takes up a tool commonly associated with the sciences - the experiment - and rethinks its role. In doing so, Fitzgerald offers a sociological analysis of the role of experiment, and how this paradigmatic building block for the creation of scientific knowledge might be accessible to other disciplines too. In arguing that experiment might be taken out into other disciplines, rather than being rejected by them for being excessively scientific, he suggests that the sciences do not exclusively own the idea of experimentation. For Fitzgerald, the experiment should be seen as having life elsewhere, in what he calls the logic of the inter/experiment, where the virgule suggests connection as well as difference. In conclusion, Fitzgerald contends that extended experiments might happen in the humanities and the social 
sciences, rather than the humanities and social sciences being regarded only as the place where what happens in experiments is discussed and interrogated.

\section{Speculations}

From re-thinking dichotomies and offering productive approaches that move beyond the usual call for interdisciplinarity, the authors in our next section examine four productive areas for ScienceHumanities research. In the first article of this section, literary scholar David Clifford offers a personally-written case study on the evolution of language. In the process, he traces a scientifically-grounded narrative that culminates in a world we describe using the humanities. He also highlights in his own practice the necessity of combining the sciences with the humanities to come to an understanding of past and present human interactions. As with the contributions in the first section, Clifford challenges the longstanding presumption that science sits opposed to the humanities and, in doing so, he speculates on a new bilateral relationship between these fields. His argument defends the humanities against contemporary accusations of a lack of utility (sometimes made by the sciences). Instead, Clifford reveals that it is only through aligning humanities knowledge with scientific explanation that we can come to an understanding of human societies. The next article is similarly focussed on the challenges to traditional disciplinarity, but looks more to our contemporary moment than to pre-history. In it, historian Chris Otter examines the rise of what he calls encapsulation: the ever-increasing confinement of human existence within various forms of capsules from our own cars and homes to our air-conditioned indoor public spaces and containerised transportation. For Otter, this phenomenon can only be addressed by multiple disciplines working in tandem; thinking sociologically, historically, technologically, scientifically, and medically. He argues that the diversity and extension of encapsulation requires us to draw rigorously from the knowledge base of varied disciplines. As Otter's article suggests, it is only through disciplinary collaboration that we can make visible the capsules we all live within, so that they can then become the proper object of research. This is an important argument about the power of transdisciplinary work to uncover new research subjects and to place before us those aspects of our lives that require interrogation. Otter argues, in other words, that it is not just that collaborative working is necessary to face what we understand as grand societal challenges but that it is also necessary to discover what those challenges might be.

Like Otter, Bradon Smith looks to the past and to the present to speculate on how the Energy Humanities might reconcile the sciences with the humanities in the future. In thinking about energy and the varied speculations surrounding its discourse, Smith explores how scholars can harness long-standing humanities methods in new domains. In particular, Smith argues persuasively that a complex understanding of narrative is vital for interrogating the imagined futures that need to be constructed if we are to address the energy challenges of both the present and the future. In the process, Smith's contribution suggests that, for example, work on petrocultures and climate change are excellent examples of interactions with the sciences that need to be continually reconsidered and evaluated. In the next article, working from the perspective of another form of humanities collaboration, Dan Cordle offers a lively and nuanced examination of the Nuclear Humanities and how it reassesses the impacts of entering the nuclear age. Using Dexter Masters' novel The Accident (1955) as his focus, and touching on ideas of risk and accident, Cordle addresses a fundamental question for the ScienceHumanities: how are different kinds of expertise constructed and how do they function? Like the other contributions to this special issue, Cordle challenges 
assumptions; in this case, the commonplace that literary studies have a more precarious claim to legitimacy than sociology in charting how modes of knowing shift between professional and cultural perceptions of science. Cordle cogently argues that the ScienceHumanities offers a unique position from which to understand the impact of diverse technological developments upon human identity. As Cordle eloquently states, in our world where science is both social and cultural, "the humanities cannot but be the ScienceHumanities".

\section{Practices}

Following Cordle's provocative argument that, in the nuclear age, the humanities are always connected to the sciences, Leah Knight, a literary scholar, and Alison Mark, a research associate in material engineering, explore the practicalities of scientists and humanists working together. Constructed as a dialogue in which they answer their own and each other's questions, their contribution engagingly traces the two authors' reflections upon disciplinary differences from personal, institutional, scholarly, and research perspectives. In doing so, they highlight similarities and differences and admit to being surprised by their own disciplinary assumptions as well as each other's. For Knight and Mark, the differences are often practical but, through their correspondence, they offer some suggestions on ways forward for future cross-disciplinary collaboration. In the next article, historian of science Charlotte Sleigh moves further into questions of transdisciplinary practice by thinking about projects that emerge from science and art collaborations. Sleigh argues that too much art framed around science remains beholden to scientific epistemologies. How, she asks, might art function to articulate something about science that removes the shackles of scientific power and authority over its reception? One way is to work in spaces not previously associated with science or with art. Sleigh's incisive reading of present practices invites us to reflect on how artful science is, and in the most fine-grained way to examine the nature of that artfulness, rather than using it as a superficial category to enhance the wonder of scientific achievement. For Sleigh, Art and Science (A\&S) offers ways to attend to things, agencies and actions that are less accessible to Science and Technology Studies. By "creating new and strange hybrids" through transdisciplinary working with artists, A\&S might offer one dynamic approach to answering the question of the artfulness of science and to doing the ScienceHumanities in practice.

Third in the practices section is the work of medieval literary scholar Janine Rogers, who calls for a more plural humanities. Like the authors of the prelude to this special issue, Rogers sees this as essential to resisting disciplinary hierarchies as well as the destructive binaries explored by Belling and Kirchhofer and Augusick. In doing so, Rogers turns her critical gaze to the construction of knowledge in the medieval world to show that the ScienceHumanities has a connection to the plurality and lack of division found there. For Rogers, the interrogation of medieval knowledge reminds us that differences between the disciplines are not natural but instead the creation of dominant ideologies. Working with medieval knowledge can reveal some of the things we have forgotten. It can also remind us of productive ways of working that we no longer employ. For Laurence Talairach, in the final article of this section, productive working across the disciplines was also at the heart of a project she describes in personal terms. Like Sleigh, Talairach turns to art - in this case film - to unpick the benefits and challenges of transdisciplinary collaboration. As a former medical student and as a literary scholar, Talairach offers a fascinating account of her work with medical museums. In the process, she offers fresh insight into nineteenth-century medical specimens and wax models by drawing on the aesthetic practices of the humanities 
rather than the insight or conservational expertise of the sciences. The literary and filmic expertise that enabled this collaboration shows how collaborations can (and, we suggest, should) be directed by the humanities, rather than the more common structure of scientific leadership. Ultimately Talairach's project highlights the need for rigorous evaluative methodologies to see and articulate the new knowledge that emerges in the spaces between disciplines - that is, a way of giving articulation to transdisciplinarity in its most innovative forms.

\section{Coda}

The special issue is brought to a conclusion by literary scholar and critical theorist Robert Mitchell's coda on smartness and the contemporary university. Mitchell offers a nuanced reading of the university as demanding the kinds of slow research that smartness (commonly defined by various smart systems and technologies) does not always value, and sometimes works to undermine. Mitchell's view is not an easy rejection of smartness but instead argues that to understand smartness and its scientific technologies properly we must do so with the eye of the humanities scholar. He notes that this critical eye can offer a properly questioning role and, furthermore, that such an approach can maintain a complex understanding of temporality, which displaces the amnesia effect of some contemporary scientific technologies. The slowing down required by acts of remembering extended time enables a balance to be struck between the reflective and the active, and also offers an opportunity to consider the values and goals of smart technologies which have themselves no sense of an ending and no clear commitments.

Mitchell's coda, and the other articles in the special issue, remind us of the key intellectual and political ideas at stake in thinking with and through the ScienceHumanities. We are reminded that how we understand and how we control the challenges of the present and the future is something that cannot be left to the sciences alone. There are very few uncontaminated spaces where productive work can occur. Too many locations, whether actual or intellectual, are already in the hands of one discipline or another. The ScienceHumanities, therefore, provides a new space that can initiate and extend profitable modes of working, rather than accepting that scientific epistemologies are always more likely to give access to the truth. It offers an opportunity for the humanities to become more active in extending scientific practices into new arenas, rather than merely commenting upon scientific developments as they occur. Finally, as Mitchell concludes and as is true across all the articles gathered here, this essential work requires time: time to develop and conduct it, yes, but also a proper sense of temporality that acknowledges the past and can look forward to the future. 